\title{
Sieve bootstrapping in the Lee-Carter model
}

Citation for published version (APA):

Heinemann, A. (2013). Sieve bootstrapping in the Lee-Carter model. Maastricht University, Graduate School of Business and Economics. GSBE Research Memoranda No. 069 https://doi.org/10.26481/umagsb.2013069

Document status and date:

Published: 01/01/2013

DOI:

10.26481/umagsb.2013069

Document Version:

Publisher's PDF, also known as Version of record

\section{Please check the document version of this publication:}

- A submitted manuscript is the version of the article upon submission and before peer-review. There can be important differences between the submitted version and the official published version of record.

People interested in the research are advised to contact the author for the final version of the publication, or visit the DOI to the publisher's website.

- The final author version and the galley proof are versions of the publication after peer review.

- The final published version features the final layout of the paper including the volume, issue and page numbers.

Link to publication

\footnotetext{
General rights rights.

- You may freely distribute the URL identifying the publication in the public portal. please follow below link for the End User Agreement:

www.umlib.nl/taverne-license

Take down policy

If you believe that this document breaches copyright please contact us at:

repository@maastrichtuniversity.nl

providing details and we will investigate your claim.
}

Copyright and moral rights for the publications made accessible in the public portal are retained by the authors and/or other copyright owners and it is a condition of accessing publications that users recognise and abide by the legal requirements associated with these

- Users may download and print one copy of any publication from the public portal for the purpose of private study or research.

- You may not further distribute the material or use it for any profit-making activity or commercial gain

If the publication is distributed under the terms of Article $25 \mathrm{fa}$ of the Dutch Copyright Act, indicated by the "Taverne" license above, 


\section{Maastricht University}

Alexander Heinemann

Sieve bootstrapping in the Lee-Carter model

RM/13/069

\section{GSBE}

Maastricht University School of Business and Economics

Graduate School of Business and Economics

P.O Box 616

NL- 6200 MD Maastricht

The Netherlands 


\section{Sieve Bootstrapping in the Lee-Carter Model}

Alexander M. Heinemann

a.heinemann@student .maastrichtuniversity.nl

October 31, 2013 


\begin{abstract}
This paper studies an alternative approach to construct confidence intervals for parameter estimates of the Lee-Carter model. First, the procedure of obtaining confidence intervals using regular nonparametric i.i.d. bootstrap is specified. Empirical evidence seems to invalidate this approach as it indicates strong autocorrelation and cross correlation in the residuals. A more general approach is introduced, relying on the Sieve bootstrap method, that includes the nonparametric i.i.d. method as a special case. Secondly, this paper examines the performance of the nonparametric i.i.d. and the Sieve bootstrap approach. In an application to a Dutch data set, the Sieve bootstrap method returns much wider confidence intervals compared to the nonparametric i.i.d. approach. Neglecting the residuals' dependency structure, the nonparametric i.i.d. bootstrap method seems to construct confidence intervals that are too narrow. Third, the paper discusses an intuitive explanation for the source of autocorrelation and cross correlation within stochastic mortality models.
\end{abstract}




\section{Acknowledgements}

The research to be presented in this article has been made possible thanks to a grant from the Marble project. Moreover, the author would like to thank Eric Beutner for his constructive comments and suggestions during the preparation of the paper and for his very careful reading. 


\section{Contents}

1 Introduction 1

2 Confidence Intervals in the Lee-Carter Model 1

2.1 Lee-Carter Model . . . . . . . . . . . . . . . . . . . . . . . . . . . . . . . . 1

2.2 Nonparametric i.i.d. Bootstrap . . . . . . . . . . . . . . . . . 2

2.3 Autocorrelation and Cross-Correlation Findings . . . . . . . . . . . . . 2

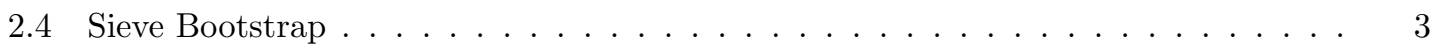

3 Comparing Bootstrap Intervals 4

4 Discussion and Conclusion $\quad 6$ 


\section{Introduction}

Over the last century, there has been a strong decline in mortality rates of developed countries. This trend poses a challenge not only for insurance companies, but also for planners of public retirement systems. Models that incorporate a stochastic component seem to be appealing instruments, because they allow to construct confidence intervals for model parameters and, more important, they return mortality rate predictions. The most widely spread stochastic mortality model is the Lee-Carter model that is employed today by the U.S. Bureau of Census and by the United Nations (Girosi and King, 2007). Its approach is based on singular value decomposition and is determined by three parameter vectors. Generally, one is not only interested in parameter point estimates, but rather in confidence intervals when fitting a mortality model to a given data set. To construct confidence intervals, a common approach relies on resampling. In the literature, the standard approach to construct confidence intervals employs the nonparametric i.i.d. bootstrap method. Applying this method, one implicitly assumes residual independence. Some researchers have already raised serious doubts regarding the method's applicability after having found autocorrelation and cross-correlation in the residuals.

This paper proposes an alternative approach to construct confidence intervals that are based on the Sieve bootstrap method. The Sieve bootstrap method is a technique that permits autocorrelated errors. It is the purpose of this paper to investigate the impact on confidence intervals when correlation is taken into account.

The remaining part of the paper is organized as follows: Section 2 presents a guideline how confidence intervals are constructed under the standard and the alternative approach. In Section 3, both methods are applied to a Dutch data set and analyzed on a comparative basis. Section 4 concludes the paper.

\section{Confidence Intervals in the Lee-Carter Model}

\subsection{Lee-Carter Model}

Lee and Carter (1992) were pioneers in the field of stochastic mortality models. In their original model, they set $m_{x, t}$, the central death rates at age $x$ in year $t$, in a logarithmic relation to its parameters. The model can be written as follows:

$$
\ln \left(m_{x, t}\right)=a_{x}+b_{x} k_{t}+\varepsilon_{x, t}, x=0, \ldots, X, t=1, \ldots, T .
$$

It is note-worthy that in this model $a_{x}$ and $b_{x}$ are age-specific constants that do not vary with time. In contrast, the parameter $k_{t}$ is a time-varying index. The model is particularily convenient due to its easy-to-interpret parameters:

- $a_{x}$ is an age-specific constant; $\exp \left(a_{x}\right)$ seen as a function of $x$ is the general shape of the mortality schedule

- $k_{t}$ is the time-varying overall mortality index

- $b_{x}$ is the age-specific sensitivity to the time-varying index 
see, for example, Brouhns, Denuit and Keilegom (2005). Moreover, the model is quite popular as the computational effort is rather small compared to other models. Parameter estimates can be found by minimizing the mean squared error:

$$
\min \quad M S E=\sum_{x, t}\left[\ln \left(m_{x, t}\right)-a_{x}-b_{x} k_{t}\right]^{2} .
$$

As the alert reader might have noticed, there are infinitely many possibilities for $a_{x}, b_{x}$ and $k_{t}$. Suppose one has found $a_{x}^{*}, b_{x}^{*}$ and $k_{t}^{*}$ that satisfy (2), it is easy to verify that $a_{x}^{\prime}=a_{x}^{*}$, $b_{x}^{\prime}=c b_{x}^{*}$ and $k_{t}^{*}=\frac{k_{t}^{*}}{c}$ for $c \in \mathbb{R} \backslash\{0,1\}$ also solve the set of equations (see, for example, Renshaw and Haberman (2003)). To ensure identification of the parameters, Lee and Carter impose the following constraints:

$$
\sum_{t} k_{t}=0 \quad \sum_{x} b_{x}=1 .
$$

The parameter $a_{x}$ can be estimated by the mean log-rate for each age group $x$, whereas estimates for $b_{x}$ and $k_{t}$ can be found by a computational procedure called singular value decomposition, see Lee and Carter (1992). To construct confidence intervals for these parameter estimates one relies on bootstrapping, which is addressed in the subsequent sections.

\subsection{Nonparametric i.i.d. Bootstrap}

To construct confidence intervals for the parameters $a_{x}, b_{x}$ and $k_{t}$, many researchers employ Koissi et al. (2006)'s approach that is based on resampling residuals (with replacement). Using the mortality model's parameter estimates $\hat{a}_{x}, \hat{b}_{x}$ and $\hat{k}_{t}$, one finds the residuals by subtracting the fitted value, $\hat{a}_{x}+\hat{b}_{x} \hat{k}_{t}$, from the observed log-central death rates, $\ln \left(m_{x, t}\right)$. Koissi et al.'s procedure is frequently called nonparametric i.i.d. bootstrap. According to this technique one finds confidence intervals by following the steps below:

- Step 1

resample the residuals (with replacement) and generate $N$ bootstrap sample tables

$$
\text { Lee-Carter: } \ln \left(m_{x, t}^{n}\right)=\hat{a}_{x}+\hat{b}_{x} \hat{k}_{t}+\varepsilon_{x, t}^{n}
$$

- Step 2

for each table $n$ : reestimate the model parameters, i.e. $\hat{a}_{x}^{n}, \hat{b}_{x}^{n}$ and $\hat{k}_{t}^{n}$

- Step 3

plot the $N$ times reestimated parameters in histograms and select $(1-\alpha) \%$ - intervals

It is noteworthy that the nonparametric i.i.d. bootstrap relies on the empirical distribution function of the residuals and assumes that they are i.i.d.. If this assumption is not given, the approach is not valid and thus produces inadequate results.

\subsection{Autocorrelation and Cross-Correlation Findings}

In multivariate time series econometrics one distinguishes between autocorrelation and crosscorrelation. Given panel data, autocorrelation is the correlation across the time axis, whereas cross-correlation is the correlation between different groups. In the case of stochastic mortality 
models, autocorrelation is the correlation along the time-axis and cross-correlation is the correlation between different age groups. With respect to the latter, it is common to differentiate between cross-correlation across age $(x)$ and cross-correlation across the cohort $(t-x)$. Koissi et al. (2006) investigated the residuals' correlation structure of stochastic mortality models by plotting contour maps. After fitting the Lee-Carter model to Danish, Finnish, Norwegian and Swedish data sets, they analyzed the residual matrices graphically and observed cluster regions indicating a certain dependency structure. Renshaw and Haberman (2005) also observed such a non-random pattern in the (standardized) residuals when applying the Lee-Carter model to a UK data set. They proposed to add a second bilinear term to the Lee-Carter model hoping to capture some correlation persistent in the Lee-Carter residuals across the cohort. Neither Renshaw and Haberman nor Koissi et al. reported results from a test on zero-autocorrelation or zero-cross-correlation across the natural axes (age, time and cohort). Dowd et al. (2010) addressed autocorrelation in stochastic mortality models in their goodness-of-fit analysis in application to an English and Welsh data set. Among others, they have investigated the Lee-Carter model and Renshaw and Haberman's extension for autocorrelation and cross-correlation. They conclude that neither of the residual matrices has independent observations. It seems that autocorrelation and crosscorrelation is a recurring issue in the Lee-Carter model, but also in other stochastic mortality models. As stated in the previous subsection, when correlation is present in the residual matrix, the nonparametric i.i.d. bootstrap technique is not a valid approach. Therefore, another method is needed that incorporates the residual dependence structure when constructing confidence intervals.

\subsection{Sieve Bootstrap}

In the following subsection, an alternative bootstrap approach is proposed, that adjusts for the correlation structure of the errors. Bühlmann $(1997,1998)$ designed the Sieve bootstrap technique for univariate time-series. This technique can be adopted to the case of multivariate time-series, i.e. vector-autoregressive models. The underlying idea is to unravel the dependence structure of the error terms by fitting an auto-/cross-correlation model and then resample (with replacement) from the new model's residuals. Having obtained the Lee-Carter parameters estimates, $\hat{a}_{x}, \hat{b}_{x}$ and $\hat{k}_{t}$, one needs to determine the residual vectors, $\boldsymbol{\varepsilon}_{t}=\ln \left(\mathbf{m}_{t}\right)-\hat{\mathbf{a}}-\hat{\mathbf{b}} \hat{k}_{t}, t=1, \ldots, T$, before one can construct confidence intervals. Here $\mathbf{m}_{t}=\left(m_{1, t}, \ldots, m_{X, t}\right)^{\prime}, t=1, \ldots, T, \hat{\mathbf{a}}=\left(\hat{a}_{1}, \ldots, \hat{a}_{X}\right)^{\prime}$ and $\hat{\mathbf{b}}=\left(\hat{b}_{1}, \ldots, \hat{b}_{X}\right)^{\prime}$, where ' denotes the transpose of a vector. Now we proceed as follows:

- Step 1

fit an appropiate auto-/cross-correlation model to the residual matrix

- Step 2

generate $N$ bootstrap residual tables, i.e. $\varepsilon_{t}^{n}$, by resampling the errors of the auto-/crosscorrelation model

- Step 3

With the $N$ bootstrap residual vectors tables create $N$ bootstrap sample tables

$$
\text { Lee-Carter: } \ln \left(\mathbf{m}_{t}^{n}\right)=\hat{\mathbf{a}}+\hat{\mathbf{b}} \hat{k}_{t}+\varepsilon_{t}^{n}
$$

- Step 4

for each table n: reestimate the model parameters, i.e. $\hat{a}_{x}^{n}, \hat{b}_{x}^{n}$ and $\hat{k}_{t}^{n}$ 
- Step 5

plot the $N$ reestimated model parameters in histograms and select $(1-\alpha) \%$ - intervals

If the error terms are i.i.d., the auto-/cross-correlation model will generate insignificant parameters such that the proposed Sieve bootstrap method will produce (asymptotically) the same results as the nonparametric i.i.d. bootstrap method. When the error terms are dependent, the results of the Sieve bootstrap and of the nonparametric i.i.d. bootstrap will be different. The crux is to find an appropriate auto-/cross-correlation model for the Sieve bootstrap method. Usually, Sieve bootstrap is applied to a univariate residual series leading to a single equation that explains the autocorrelation. In the case of stochastic mortality models, one deals with a residual matrix, whose autocorrelation and cross-correlation cannot be explained by a single equation but rather a system of equations with interacting terms.

\section{Comparing Bootstrap Intervals}

In order to compare the nonparametric i.i.d. and the Sieve bootstrap method, the Lee-Carter model was applied to a Dutch data set freely provided by the Human Mortality Database (2013). To invalidate any small-sample-auto-/cross-correlation argument, a large sample was selected ranging from 1850 to 2009 with age groups from 0 to 98 (160x99 matrix). If neither autocorrelation nor cross-correlation between the errors exists, we would expect the Sieve bootstrap's auto-/crosscorrelation model to have predominantly insignificant parameters. In that case, the nonparametric i.i.d. and the Sieve bootstrap method are likely to result in similar confidence intervals for the parameters $a_{x}, b_{x}$ and $k_{x}$. In contrast, if autocorrelation and/or cross-correlation are persistent, we expect the Sieve bootstrap method to result in wider confidence intervals, whereas the nonparametric i.i.d. bootstrap method underrates the interval's width by neglecting the residual dependence structure.

For the Sieve bootstrap method, a system of simultaneous equations, dominated by first-order autocorrelation terms and cross-correlation between neighboring age groups (age and cohort) was found to be an appropriate auto-/cross-correlation model (using the Bayesian information criterion). More than two third of the model's parameters were significant at a $1 \%$-significance level indicating a strong dependence structure.

Creating 3001 bootstrap samples under the nonparametric i.i.d. and Sieve bootstrap method and reestimating the model parameters one is able to select appropriate $1-\alpha \%$ confidence intervals. Because the Lee-Carter model incorporates $2 * 99+160=258$ parameters, it is illusory to compare the bootstrap confidence intervals for every single parameter. Instead, the subsequent analysis focuses on nine representative parameters: $a_{x}, b_{x}$ and $k_{t}$ with $x \in\{0 ; 50 ; 90\}$ and $t \in\{1850 ; 1900 ; 2009\}$ with comments to the average performance incorporating the other 249 parameters.

Figure 1 illustrates the bootstrap confidence intervals of the parameter $a_{x}$ for three different age groups. On the left side, the confidence intervals constructed by the nonparametric i.i.d. bootstrap method are presented, whereas on the right hand side the Sieve bootstrap confidence intervals are shown. For all three age groups, as well as for the other 95 age groups, it can be inferred, that both methods produce a mean that is not statistically different from each other. The confidence intervals, however, differ substantially in their widths. For the age group 0 , the standard deviation 


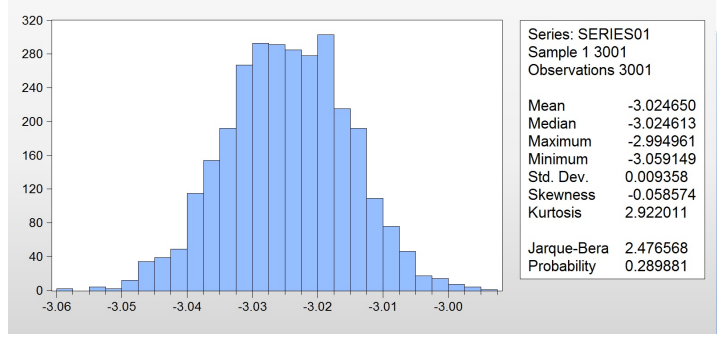

(a) Age group 0: nonparametric i.i.d. bootstrap

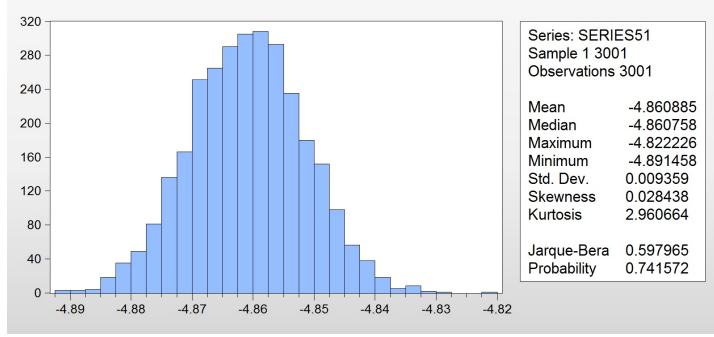

(c) Age group 50: nonparametric i.i.d. bootstrap

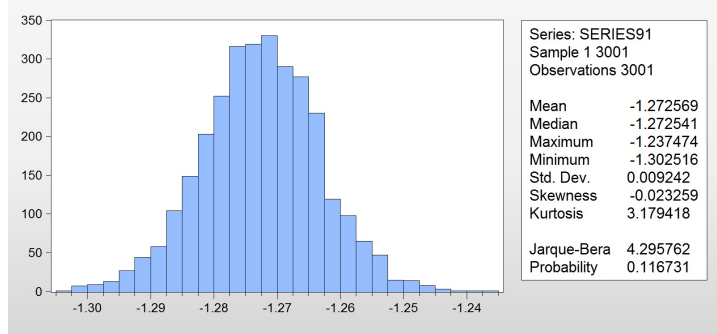

(e) Age group 90: nonparametric i.i.d. bootstrap

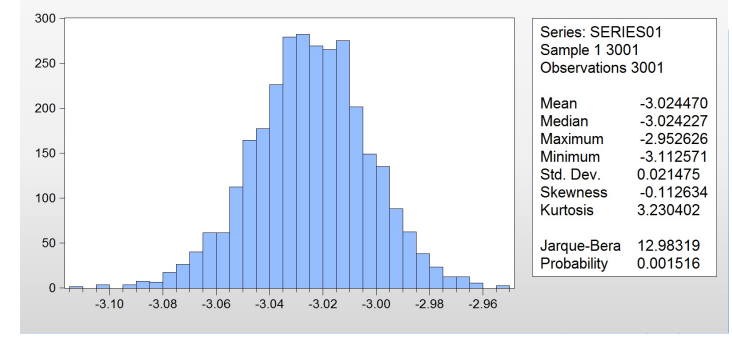

(b) Age group 0: Sieve bootstrap

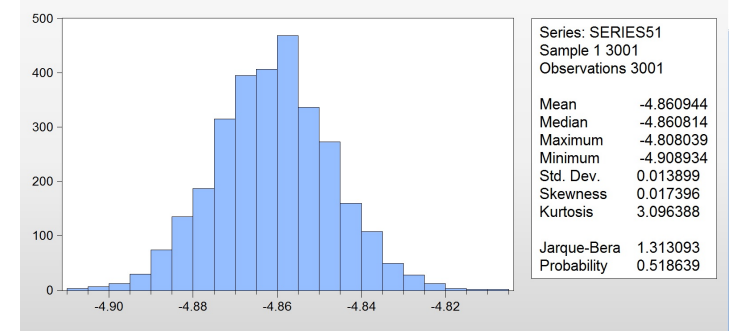

(d) Age group 50: Sieve bootstrap

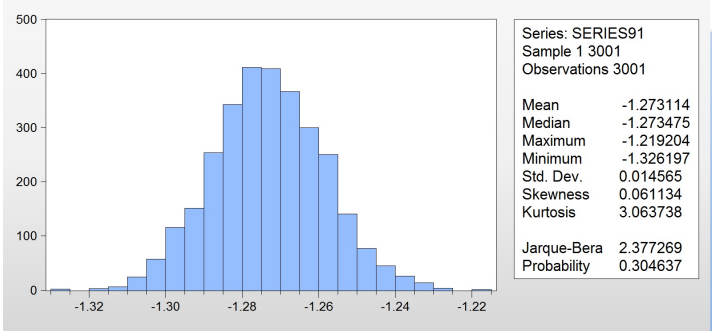

(f) Age group 90: Sieve bootstrap

Figure 1: $a_{x}$ confidence intervals

of the Sieve bootstrap confidence interval is $130 \%$ larger than the confidence interval constructed under the nonparametric i.i.d. bootstrap method. For the age group 50, the standard deviation of the Sieve bootstrap confidence interval is $49 \%$ larger and for the age group 90, the standard deviation of the Sieve bootstrap confidence interval is 58\% larger. Taking all age groups into consideration, the standard deviation of the Sieve bootstrap confidence interval is on average $59 \%$ larger than the confidence interval constructed under the nonparametric i.i.d. bootstrap method.

Regarding the parameter $b_{x}$, one finds similar results. Again, the means resulting from the different methods are not statistically different in the age groups, however the methods differ in the widths. On average, the standard deviation of the Sieve bootstrap confidence interval is $56 \%$ larger than the confidence interval constructed under the nonparametric i.i.d. bootstrap method. Figure 2 illustrates the bootstrap confidence intervals for three different age groups. For age group 0, 50 and 90 the respective difference in the standard deviations are $128 \%, 43 \%$ and $46 \%$ respectively.

With respect to the parameter $k_{t}$, again the nonparametric i.i.d. bootstrap method seem to understate the confidence interval width, though the mean tend to be the same. Figure 3 shows the bootstrap confidence intervals for three different years. For the starting year 1850, the standard deviation of the Sieve bootstrap confidence interval is $65 \%$ larger than the confidence interval 


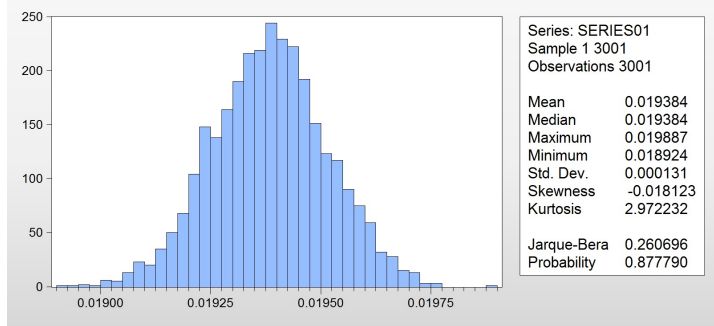

(a) Age group 0: nonparametric i.i.d. bootstrap

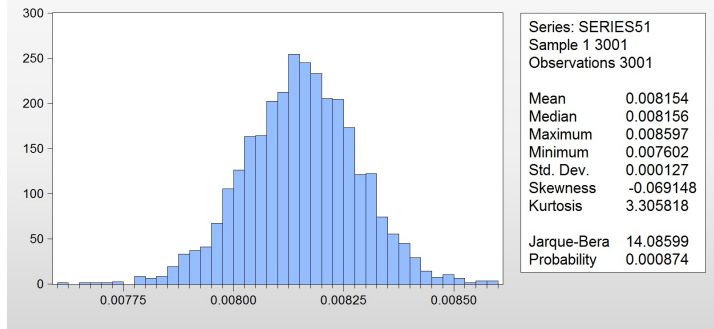

(c) Age group 50: nonparametric i.i.d. bootstrap

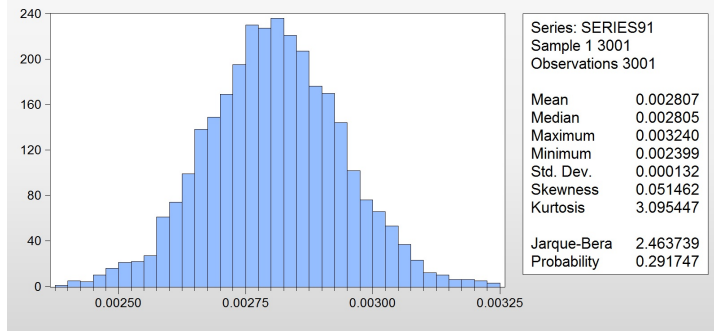

(e) Age group 90: nonparametric i.i.d. bootstrap

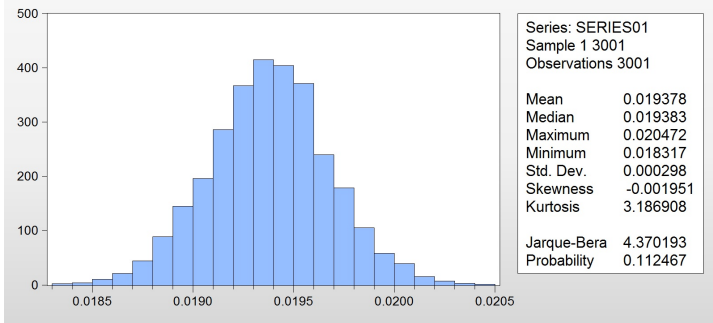

(b) Age group 0: Sieve bootstrap

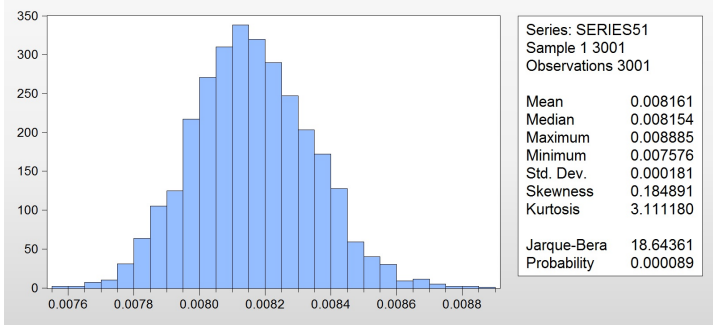

(d) Age group 50: Sieve bootstrap

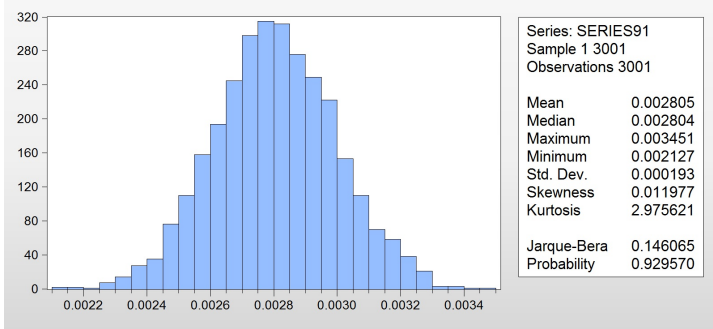

(f) Age group 90: Sieve bootstrap

Figure 2: $b_{x}$ confidence intervals

constructed under the nonparametric i.i.d. bootstrap method. For the year 1900, the standard deviation of the Sieve bootstrap confidence interval is $123 \%$ larger and for end year 2009, the standard deviation of the Sieve bootstrap confidence interval is $111 \%$ larger. Also in the other years the nonparametric i.i.d. bootstrap method underestimates the confidence interval width: on average, the standard deviation of the Sieve bootstrap confidence intervals are $113 \%$ larger.

The previous analysis clearly shows that disregarding the residual dependency structure by applying the nonparametric i.i.d. bootstrap method is precarious. The nonparametric i.i.d. bootstrap method may produce confidence intervals that are too narrow. In the Dutch application, the nonparametric i.i.d. bootstrap method underestimated the standard deviation for the parameter estimates $\hat{a}_{x}, \hat{b}_{x}$ and $\hat{k}_{t}$ on average by $59 \%, 56 \%$ and $113 \%$, respectively. Taking autocorrelation and cross-correlation into account by applying the Sieve bootstrap method results in much larger confidence intervals, that seem to be more accurate.

\section{Discussion and Conclusion}

Not only the previous analysis, but also the persistency of autocorrelation and cross-correlation in different studies suggests that correlation among residuals is a recurrent issue in stochastic 


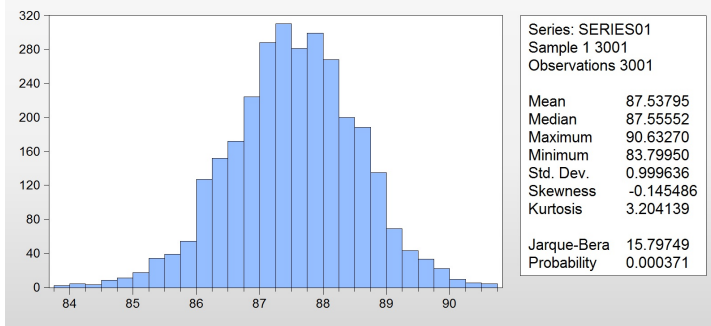

(a) Age group 0: nonparametric i.i.d. bootstrap

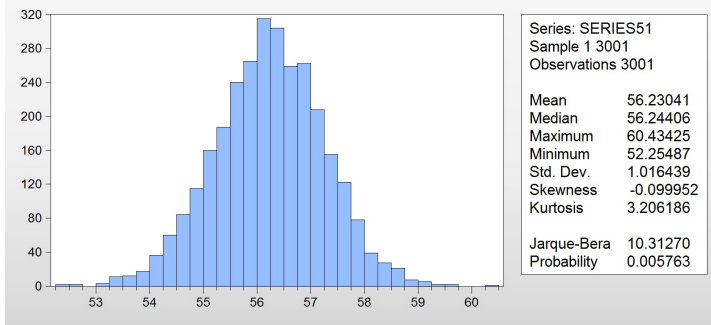

(c) Age group 50: nonparametric i.i.d. bootstrap

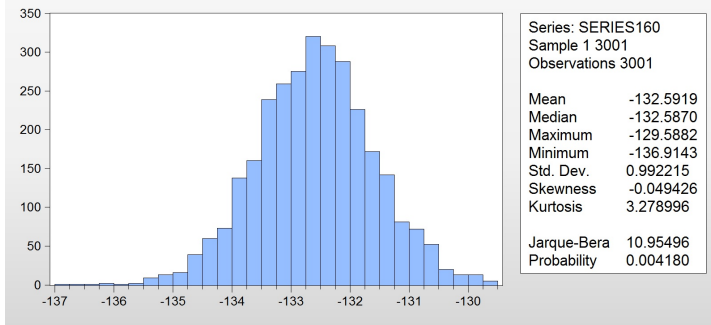

(e) Age group 90: nonparametric i.i.d. bootstrap

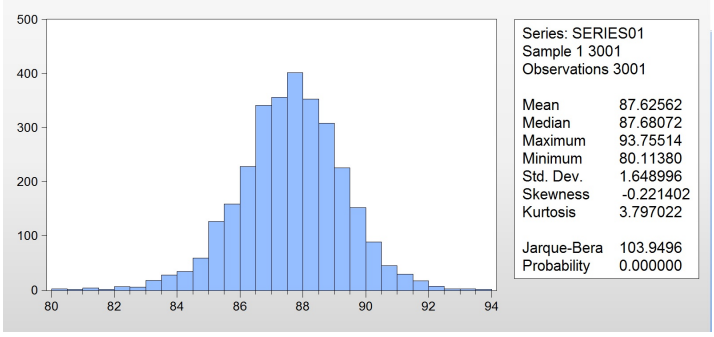

(b) Age group 0: Sieve bootstrap

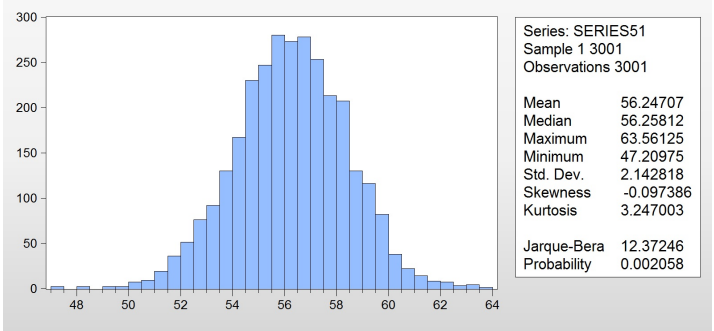

(d) Age group 50: Sieve bootstrap

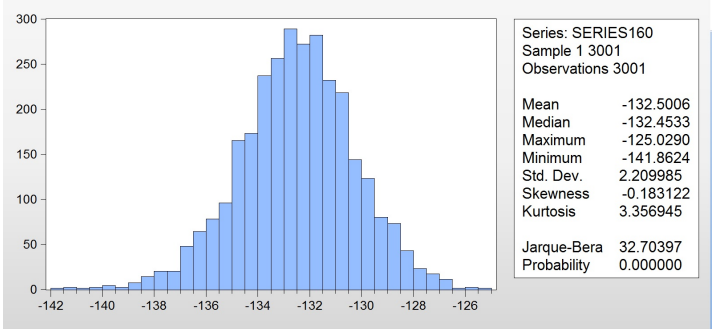

(f) Age group 90: Sieve bootstrap

Figure 3: $k_{t}$ confidence intervals

mortality models that needs to be taken into account. Regarding confidence intervals for model parameters, it was shown that the method of nonparametric i.i.d. bootstrap, that disregards the residual dependence structure, tends to underestimate the intervals' width. The Sieve bootstrap method was proposed as an alternative approach that is also valid when there exists residual autocorrelation and crosscorrelation. For the Dutch data set, the method generated confidence intervals with similar means, but larger standard deviations. The core of this approach is to find an appropriate auto-/crosscorrelation model, that may be cumbersome. For the selected Dutch data, it is remarkable that primarily first-order autocorrelation and cross-correlation between neighboring age groups was detected and found to be highly significant. This specific auto-/crosscorrelation result may be a consequence from following (approximately) the same group of individuals over time. To illustrate the argument, a Lexis Plane is plotted in Figure 4 with time on the horizontal axis and age on the vertical axis.

Each colored line presents an individual; All colored lines create a $45^{\circ}$ angle with the x-axis. The $45^{\circ}$ angle comes from the fact that an increase in time by $\tau$ goes along with an increase in age by $\tau$. The individuals' lines do not coincide, but are parallelly shifted due to different dates of birth. The highlighted square contains the group of people aged $x$ in the year $t$ and is defined as $[t, t+1) \times[x, x+1)$. In the given Lexis Plane all seven individuals are observed in the calendar year $t$ when aged $x$. As time passes, these individuals will be observed in the calendar 


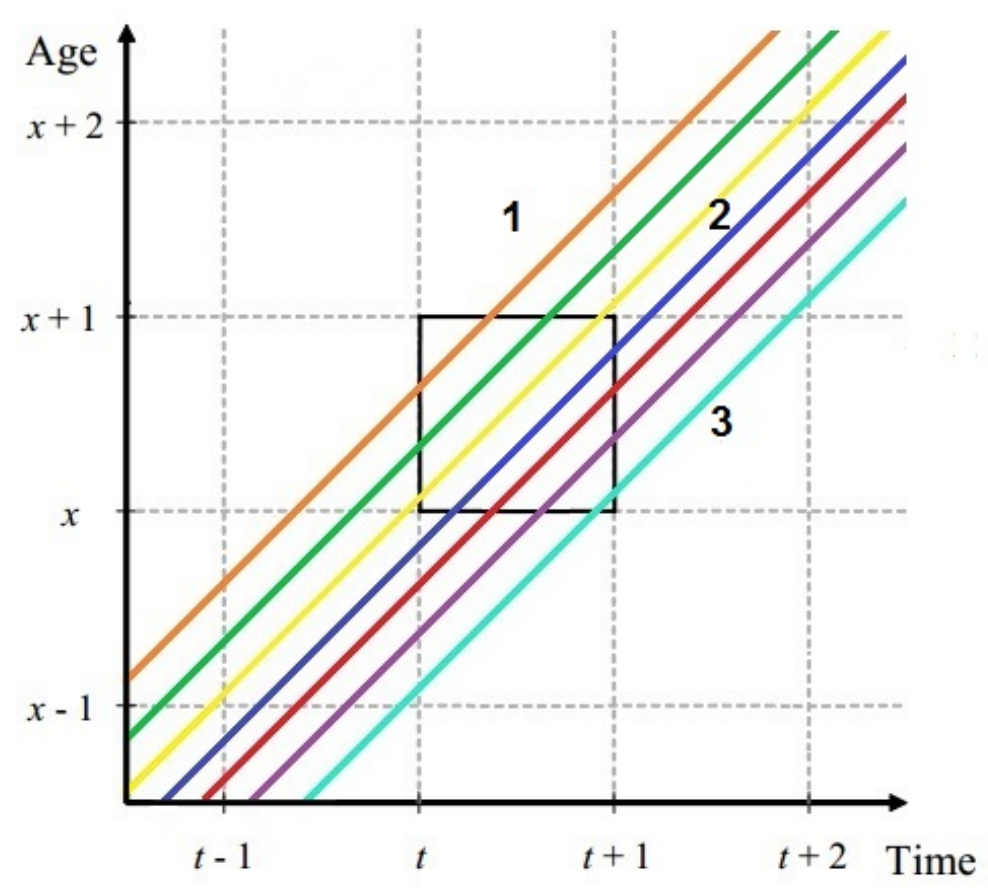

Figure 4: Lexis Plane

year $t+1$ when they are aged $x+1$, indicated by square number 2 . Moreover, approximately half will be observed in the square 1 and by analogue arguments approximately half are also observed in square 3. Therefore, by following the same (sub-)groups of individuals, one could actually expect first-order autocorrelation and cross-correlation along the age- and the cohort-axis. This reasoning is coherent with the observed patterns, however, further investigation will be required to draw a conclusion.

In summary, autocorrelation and cross-correlation, whether they have a theoretical explanation or not, play an important role in constructing confidence intervals for the Lee-Carter parameters. The current method of nonparametric i.i.d. bootstrap seem to understate the width of the confidence intervals as it omits the residual dependency structure. Therefore, the proposed method of Sieve bootstrap, that takes autocorrelation and cross-correlation into account, is needed. This method is not restricted to the Lee-Carter model only, but can also be employed when autocorrelation and/or cross-correlation are persistent in other stochastic mortality models. 


\section{References}

Bühlmann, P. (1997). Sieve Bootstrap for Time Series. Bernoulli, 3, 123-148.

Bühlmann, P. (1998). Sieve Bootstrap for Smoothing Nonstationary Time Series. Annals of Statistics, 26, 48-83.

Brouhns, N., Denuit, M. and Keilegom, I.V. (2005). Bootstrapping the poisson Log-bilinear Regression Approach to the Construction of Projected Lifetables. Scandinavian Actuarial Journal, 3, 212-224.

Dowd, K., Cairns, A., Blake, D., Coughlan, G., Epstein, D. and Khalaf-Allah, M. (2010). Evaluating the goodness of fit of stochastic mortality models. Insurance: Mathematics and Economics, $47,255-265$.

Girosi, F. and King, G. (2007). Understanding the Lee-Carter Mortality Forecasting Method. Working paper, Harvard University, USA.

Human Mortality Database (2013). Netherlands: Complete Data Series. Retrieved from http://www.mortality.org on 10 April, 2013.

Koissi, M., Shapiro, A. and Högnäs, G. (2006). Evaluating and extending the Lee-Carter model for mortality forecasting: Bootstrap confidence interval. Insurance: Mathematics and Economics, $38,1-20$.

Lee, R. and Carter, L. (1992). Modeling and Forecasting U. S. Mortality. Journal of the American Statistical Association, Vol. 87, No. 419, 659-671.

Renshaw, A. and Haberman, S. (2005). Mortality Reduction Factors Incorporating Cohort Effects. Actuarial Research Paper, Cass Business School, London. UK.

Renshaw, A. and Haberman, S. (2003). A Parallel Generalized Linear Modelling Approach for England and Wales Mortality Projections. Journal of the Royal Statistical Society, Series C, Vol. 52, No. 1, 119-137. 